\title{
Síntese de Copolímeros em Bloco de p-Acetoxiestireno com Isopreno através de Polimerização "Viva” Via Radical Livre
}

\author{
José Carlos Moreira, Shu Hui Wang \\ Departamento de Engenharia Metalúrgica e de Materiais, USP
}

\begin{abstract}
Resumo: Neste trabalho, é mostrada a síntese de homopolímeros de p-acetoxiestireno e copolímeros diblocos de pacetoxiestireno com isopreno, utilizando um iniciador TEMPO-modificado, por um processo de polimerização "viva" via radical livre. As análises de GPC indicaram a formação de copolímeros em bloco com massas molares e polidispersão mais altas quando comparadas aos dos homopolímeros correspondentes. Os copolímeros em bloco poli(p-acetoxiestirenob-isopreno) foram preparados com controle de composição e massa molar até 94500 u.m.a. As análises de DSC mostraram que a transição vítrea do bloco de p-acetoxiestireno foi de 5 a $18{ }^{\circ} \mathrm{C}$ mais baixa que aquelas obtidas para os homopolímeros correspondentes. A transição vítrea do bloco de isopreno apresentou valores similares àqueles dos homopolímeros de poliisopreno $\left(-67 \mathrm{a}-55^{\circ} \mathrm{C}\right)$.
\end{abstract}

Palavras-chave: Copolímero em bloco, p-acetoxiestireno, isopreno, síntese.

\section{Synthesis of Block Copolymers from p-Acetoxystyrene and Isoprene by “Living” Free Radical Polymerization}

Abstract: In this work we show the synthesis of homopolymers and diblock copolymers of p-acetoxystyrene and isoprene by using TEMPO-modified free radical initiator. The GPC analyses have shown an increase in the molar masses and in the polydispersity of the copolymers in comparison with their corresponding homopolymer. Poly(p-acetoxystyrene-bisoprene) were prepared with good control of composition and molar mass up to 94,500 a.m.u. DSC analyses have shown a glass transition temperature decrease of $5-18{ }^{\circ} \mathrm{C}$ for the poly(p-acetoxystyrene) segment in copolymers compared to that observed for the parent homopolymer. The glass transition temperature of polyisoprene segment in copolymers remained similar to those observed for the homopolymers $\left(-67\right.$ to $\left.-55^{\circ} \mathrm{C}\right)$.

Keywords: Block copolymer, p-acetoxystyrene, isoprene, synthesis.

\section{Introdução}

As técnicas de polimerização viva não são úteis somente para controlar massas molares e distribuições de massas molares de polímeros, mas também para sintetizar copolímeros em bloco ${ }^{[1]}$. Em geral, copolímeros em bloco são sintetizados por polimerizações iônicas, por abertura de anel ou por coordenação, por causa da natureza viva da terminação da cadeia polimérica. Os copolímeros em bloco típicos como poli(estireno-b-isopreno) e poli(estireno-bbutadieno) são preparados a partir de processos de polimerização aniônica, que requer monômeros e solventes altamente puros em atmosferas isentas de oxigênio e umidade. Por outro lado, a polimerização via radical livre é o método preferido dos engenheiros e químicos, para preparação de polímeros em grande escala, por causa do baixo custo e por ser mais flexível em termos de espécies de monômeros e presença de impurezas. Porém, a síntese via radical livre de copolímeros em bloco tem sido difícil devido à tendência das cadeias em propagação sofrerem terminação prematura irreversível ${ }^{[2]}$. Pesquisas nesta área, na década passada, conduziram ao desenvolvimento de téc- nicas de polimerização "viva" radicalar, com o objetivo de sintetizar copolímeros em bloco com distribuição de massa molar estreita ${ }^{[3-6]}$.

Os copolímeros em bloco são uma classe única de materiais poliméricos que sofrem separação de fase da ordem nanométrica e têm uma variedade de aplicações inclusive como dispersantes, compatibilizantes e modificadores de viscosidade.

Os monômeros de estireno substituído são muito interessantes devido à possibilidade de preparação de polímeros com propriedades fotofísicas e fotoquímicas $^{[7-8]}$. O controle da copolimerização de p-acetoxiestireno que resulta na formação de copolímeros em bloco permanece relativamente inexplorada. Assim sendo, existem poucos artigos na literatura que relatam a polimerização controlada via radical "livre" de p-acetoxiestireno ${ }^{[9-13]}$. Hawker et al. ${ }^{[4]}$ estudaram a copolimerização randômica em massa de isopreno com p-acetoxiestireno na presença do inciador 2,2,5-trimetil4-fenil-3-azahexano-3-óxi., sob atmosfera de argônio a $125^{\circ} \mathrm{C}$ por $36 \mathrm{~h}$. Os copolímeros de poli(p-acetoxiestireno-coisopreno) (PASt-co-PIP) foram caracterizados por GPC,

Autor para correspondência: Shu Hui Wang, Departamento de Engenharia Metalúrgica e de Materiais, USP, Av. Prof. Mello Moraes 2463, Cidade Universitária, CEP: 05508-900, São Paulo, SP. E-mail: wangshui@usp.br 


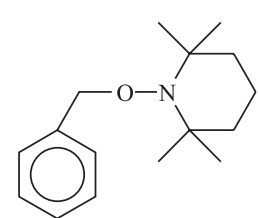

1

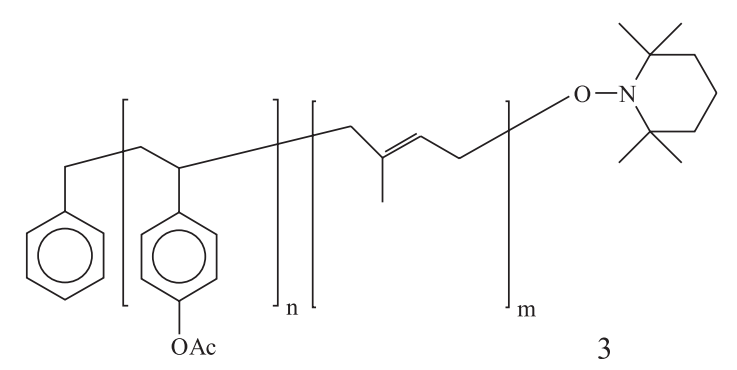

Figura 1. Estruturas químicas. (1) iniciador benzil-TEMPO, (2) macroiniciador poli(p-acetoxiestireno) e (3) poli(p-acetoxiestireno -bisopreno).

IR e RMN. Eles sintetizaram copolímeros de PASt-co-PIP bem definidos para uma faixa de composição em peso de 75 a $25 \%$ de isopreno com um bom controle da massa molar $\left(\overline{M_{n}}=17.000-20.500 \mathrm{~g} / \mathrm{mol}\right)$ e da polidispersidade $(\mathrm{PD}=$ $1,1-1,3)$. É preciso ressaltar também que a síntese de copolímero em bloco de p-acetoxiestireno com isopreno via polimerização radical livre ou outro processo de polimerização "viva" não foi reportada na literatura.

Neste trabalho, é mostrado a síntese de homopolímeros de p-acetoxiestireno (2) e copolímeros diblocos de p-acetoxiestireno com isopreno (3), através de polimerização "viva" via radical livre utilizando um iniciador TEMPO-modificado (1). As estruturas químicas aparecem representadas na Figura 1.

\section{Experimental}

\section{Materiais}

2,2,6,6-Tetrametil-1-piperidinil-oxi (TEMPO) (Aldrich), cloreto de benzilmagnésio (Aldrich) e tetra-hidrofurano anidro (THF) (Tedia) foram utilizados como recebido sem purificação adicional. Os monômeros de p-acetoxiestireno (Aldrich) e isopreno (Aldrich) foram purificados por eliminação do inibidor utilizando uma coluna básica de alumina antes da reação de polimerização.

\section{Síntese do Iniciador TEMPO-Modificado}

$\mathrm{O}$ iniciador radical livre modificado foi preparado pela reação de uma mistura de cloreto de benzilmagnésio (Aldrich) e o TEMPO a $-78^{\circ} \mathrm{C}$ por $2 \mathrm{~h}$ sob atmosfera de nitrogênio ${ }^{[14]}$. O produto (1) foi purificado por cromatografia ${ }^{[14]}$ e foi obtido um rendimento de $85 \%$. Absorções no infravermelho: $2950 \mathrm{~cm}^{-1}\left(\mathrm{CH}_{3}\right)$, $1500 \mathrm{~cm}^{-1}$ (anel aromático), $1380 \mathrm{~cm}^{-1}$ (C-O), $1030 \mathrm{~cm}^{-1}$ (N-O); espectroscopia de $\mathrm{RMN}-{ }^{1} \mathrm{H}$ a $300 \mathrm{MHz}$, em $\mathrm{CDCl}_{3}$ : $\delta 1,12$; 1,25 (s, 12H, $\mathrm{CH}_{3}$ do TEMPO), 1,29-1,54 (m, 6H, $\mathrm{CH}_{2}$ ), 4,75 (s, 2H, $\mathrm{CH}_{2}$ ), 7,17-7,30 (m, 5H, ArH); e espectroscopia de
RMN_ ${ }^{13} \mathrm{C}$ a $75 \mathrm{MHz}$, em $\mathrm{CDCl}_{3}$ : d 16,15; 19,31; 32,11; 38,79; 59,$01 ; 77,78 ; 126,25 ; 126,43 ; 127,20 ; 137,39$.

\section{Síntese de Homopolímeros de p-Acetoxiestireno}

\section{Estudos de Conversão}

Os homopolímeros de p-acetoxiestireno foram sintetizados por polimerização em massa de p-acetoxiestireno na presença de uma quantidade pré-estabelecida de iniciador TEMPOmodificado (100 mol de p-acetoxiestireno por mol de iniciador). A mistura reacional para a polimerização foi aquecida a $125^{\circ} \mathrm{C}$, sob atmosfera de nitrogênio, com amostras sendo retiradas em intervalos de tempo sucessivos.

\section{Homopolímeros Precursores}

Os homopolímeros precursores de p-acetoxiestireno também foram sintetizados por polimerização em massa de p-acetoxiestireno na presença de uma quantidade préestabelecida de iniciador TEMPO-modificado. Os homopolímeros TEMPO-terminados foram preparados com diferentes comprimentos de segmento utilizando 100, 200, e 400 mol de p-acetoxiestireno por mol de iniciador. Depois da polimerização, quando necessário o polímero formado foi diluído em clorofórmio para reduzir a viscosidade e precipitado em metanol. O produto sólido foi coletado então através de filtração a vácuo e seco a temperatura ambiente. RMN- ${ }^{1} \mathrm{H}$ a $200 \mathrm{MHz}$, em $\mathrm{CDCl}_{3}: \delta 1,39$ (s, H alifático), 2,26 (s, $\left.\mathrm{CH}_{3}\right)$, e 6,55 ( $\left.\mathrm{ArH}\right)$.

\section{Síntese de Copolímeros Diblocos}

Os copolímeros em bloco foram preparados em duas etapas. Na primeira etapa, um homopolímero precursor TEMPO-terminado foi preparado com uma massa molar pré-estabelecida. Na segunda etapa, o poli(p-acetoxiestireno) TEMPO-terminado foi dissolvido em uma pequena quantidade de THF anidro e, em seguida, isopreno foi acrescentado a esta solução sob atmosfera de nitrogênio. Então a mistura reacional foi aquecida a $125^{\circ} \mathrm{C}$ por 168 horas. Depois da polimerização, o polímero formado foi diluído em clorofórmio, quando necessário para reduzir a viscosidade, e precipitado em metanol. O produto foi coletado então através de filtração a vácuo e seco a temperatura ambiente. $\mathrm{RMN}-{ }^{1} \mathrm{H}$ a $200 \mathrm{MHz}$, em $\mathrm{CDCl}_{3}$ : $\delta$ 0,94-2,03 (m, H alifático), 2,25 (s, $\mathrm{CH}_{3}$ ), 5,12 (s, H olefínico), e 6,54 (ArH).

\section{Caracterização}

\section{Iniciador TEMPO-Modificado}

O iniciador TEMPO-modificado foi caracterizado utilizando espectrofotômetros de FTIR da Nicolet Magna-IR 560 e de RMN da Bruker de 300 MHz.

\section{Homopolímeros p-Acetoxiestireno e Copolímeros em Bloco de p-Acetoxiestireno com Isopreno}

Os homopolímeros de p-acetoxiestireno e copolímeros em bloco de p-acetoxiestireno com isopreno foram caracterizados 
utilizando espectrômetros de FTIR da Nicolet Magna-IR 560 e de RMN da Bruker de 200 MHz. Para a determinação das conversões da reação, as amostras foram transferidas diretamente da reação de polimerização para um tubo de RMN e dissolvidas em $\mathrm{CDCl}_{3}$. As porcentagens relativas de poli(p-acetoxiestireno) e p-acetoxiestireno foram determinados por integração dos picos relevantes. A composição dos copolímeros em bloco foi confirmada por análise quantitativa em solução de clorofórmio. Uma curva de calibração da banda de carbonila do grupo acetoxi $\left(1751,1 \mathrm{~cm}^{-1}\right)$ foi preparada a partir dos espectros dos homopolímeros de p-acetoxiestireno em clorofórmio a diferentes concentrações. As massas molares e polidispersão foram determinadas através de cromatografia de permeação em gel (GPC) utilizando um equipamento da Agilient 1100 equipado com colunas de Plgel em série do tipo linear com faixa de exclusão de $10^{2} \mathrm{a}$ $10^{6}$ Ângstroms e detector de índice de refração, vazão de 1 $\mathrm{ml} / \mathrm{min}$ em THF a $25^{\circ} \mathrm{C}$. Os valores de massas molares obtidos são relativos a padrões de poliestireno monodisperso. As análises de calorimetria diferencial de varredura (DSC) foram realizadas utilizando um DSC-50 Shimadzu sob atmosfera de Hélio $\left(20^{\circ} \mathrm{C} / \mathrm{min}\right)$.

\section{Resultados e Discussão}

\section{Estudos de Conversão}

Neste trabalho, os cálculos das quantidades de amostra polimerizada, de monômero residual e de porcentagem de conversão foram feitos utilizando as seguintes equações:

$$
\begin{gathered}
Q A P=\frac{\text { Área }_{-\mathrm{CH}_{3}} \times M M_{\text {Monômero }}}{3 \times M A_{\text {Hidrogênio }}} \\
Q M R=\frac{\text { Área }_{=\mathrm{CH}_{2}} \times M M_{\text {Monômero }}}{2 \times M A_{\text {Hidrogênio }}}
\end{gathered}
$$

$$
\text { Conversão }(\%)=\frac{Q A P-Q M R}{Q A P} \times 100
$$

onde QAP é a quantidade total de amostra polimerizada, QMR é a quantidade de monômero residual, Área ${ }_{-\mathrm{CH} 3}$ representa a área do pico do grupo metila $\left(-\mathrm{CH}_{3}\right)$, Área ${ }_{-\mathrm{CH}}$ representa a área do grupo metileno $\left(=\mathrm{CH}_{2}\right), \mathrm{MM}_{\text {Monômero }}$ a massa molar do monômero de p-acetoxiestireno e $\mathrm{MA}_{\text {Hidrogênio }}$ é a massa atômica do hidrogênio.

A Tabela 1 e Figura 2 mostram os valores obtidos de conversão para a síntese do homopolímero de p-acetoxiestireno a $125^{\circ} \mathrm{C}$ utilizando o iniciador benzil-TEMPO (1).

Como pode ser visto na Tabela 1 e Figura 2, a inserção do grupo acetóxi no anel aromático aumenta a velocidade de polimerização quando comparada ao estireno. O efeito do grupo substituinte elétron-aceptor sobre a velocidade de polimerização
Tabela 1. Conversão da síntese de homopolímeros de p-acetoxiestireno (PASt) em função do tempo.

\begin{tabular}{cccccc}
\hline $\begin{array}{c}\text { Área do } \\
\text { pico } \\
\left(\mathbf{C H}_{3}\right)^{\mathbf{a}}\end{array}$ & $\begin{array}{c}\text { Área do } \\
\text { pico } \\
\left(=\mathbf{C H}_{2}\right)^{\mathbf{a}}\end{array}$ & QAP & QMR & $\begin{array}{c}\text { Porcentagem } \\
\text { de } \\
\text { Conversão } \\
(\%)^{\mathbf{b}}\end{array}$ & $\begin{array}{c}\text { Tempo } \\
(\mathbf{h o r a s})\end{array}$ \\
\hline 0 & 0 & 0 & 0 & 0 & 0 \\
3,5312 & 2,0096 & 191 & 163 & 14,7 & 0,5 \\
4,0692 & 2,0080 & 217 & 166 & 23,5 & 1,0 \\
4,0209 & 2,0413 & 220 & 163 & 25,9 & 2,0 \\
6,3986 & 2,0353 & 346 & 165 & 52,3 & 3,0 \\
6,9996 & 2,0958 & 378 & 170 & 55,0 & 5,0 \\
7,6005 & 2,0823 & 410 & 169 & 58,8 & 7,0 \\
9,1985 & 2,0408 & 497 & 165 & 66,8 & 10,0 \\
- & - & - & - & $83,0^{\mathrm{b}}$ & 20,0 \\
\hline
\end{tabular}

${ }^{\mathrm{a}}$ Determinado por análises de RMN; ${ }^{\mathrm{b}}$ Determinado pela razão entre a massa inicial de monômero e a de polímero obtido.

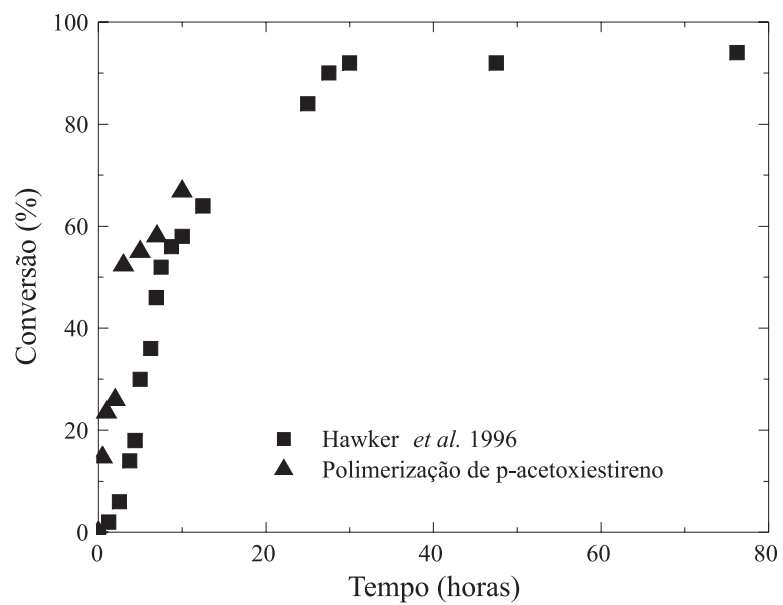

Figura 2. Evolução da conversão com o tempo para a polimerização de estireno e p-acetoxiestireno a $125^{\circ} \mathrm{C}$ utilizando o iniciador benzil-TEMPO.

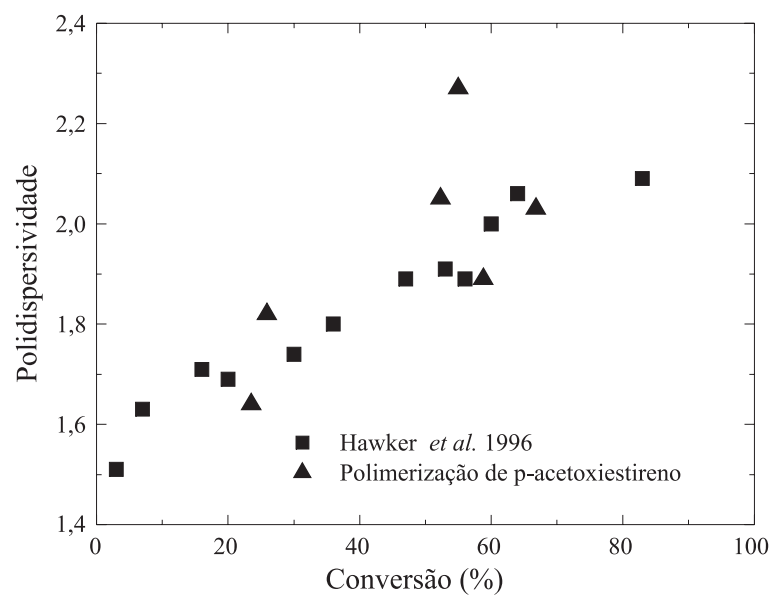

Figura 3. Polidispersidade dos polímeros em função da conversão de estireno e p-acetoxiestireno a $125^{\circ} \mathrm{C}$ utilizando o iniciador benzil-TEMPO. 
Tabela 2. Massa molar, polidispersidade e transição vítrea para poli(p-acetoxiestireno) (PASt) e poli(p-acetoxiestireno-b-isopreno) (PASt-b-PIP).

\begin{tabular}{|c|c|c|c|c|c|c|c|}
\hline \multirow{2}{*}{$\begin{array}{l}\text { Amostra } \\
\text { Copolímero }\end{array}$} & \multicolumn{2}{|c|}{$\begin{array}{l}\text { PASt bloco } \\
\text { iniciante }^{\mathrm{a}}\end{array}$} & \multirow{2}{*}{$\frac{\operatorname{Tg}\left({ }^{\circ} \mathbf{C}\right)^{\mathbf{b}}}{\text { PASt }}$} & \multirow{2}{*}{$\begin{array}{c}\text { Composição }^{c} \\
\text { ASt/IP }\end{array}$} & \multicolumn{2}{|c|}{$\begin{array}{c}\text { Copolímero em bloco de } \\
\text { PASt-b-PIPa }\end{array}$} & \multirow{2}{*}{$\frac{\mathbf{T g}\left({ }^{\circ} \mathbf{C}\right)^{\mathbf{b}}}{\text { PASt-b-IP }}$} \\
\hline & $\bar{M}_{w}$ & $\mathrm{PD}$ & & & $\bar{M}_{w}$ & PD & \\
\hline I & 17400 & 1,34 & 122,0 & $46,8 / 53,2$ & 30200 & 1,83 & $114,7 /-64,4$ \\
\hline II & 17400 & 1,34 & 122,0 & $36,8 / 63,2$ & 34700 & 1,72 & $117,4 /-62,9$ \\
\hline III & 17400 & 1,34 & 122,0 & $42,7 / 57,3$ & 33200 & 1,87 & $104,6 /-65,0$ \\
\hline IV & 34600 & 1,59 & 126,0 & $45,2 / 54,8$ & 42300 & 1,96 & $113,0 /-64,4$ \\
\hline V & 34600 & 1,59 & 126,0 & $43,7 / 56,3$ & 45000 & 1,75 & $107,7 /-62,2$ \\
\hline VI & 79200 & 1,69 & 126,3 & $53,3 / 46,7$ & 85600 & 2,34 & $116,1 /-60,4$ \\
\hline VII & 79200 & 1,69 & 126,3 & $30,8 / 69,2$ & 94500 & 1,82 & $109,1 /-63,6$ \\
\hline
\end{tabular}

Determinado por análises ${ }^{\mathrm{a}} \mathrm{GPC},{ }^{\mathrm{b}} \mathrm{DSC}$ e ${ }^{\mathrm{F}} \mathrm{FTIR}$; PD representa a polidispersidade.

radical livre "viva" mediada por nitróxido foi anteriormente observado por Barclay e colaboradores ${ }^{[1]}$.

A Figura 3 mostra o gráfico da polidispersidade em função da conversão para estireno ${ }^{[14]}$ e p-acetoxiestireno a $125^{\circ} \mathrm{C}$, utilizando o iniciador de benzil-TEMPO (1).

Em ambos os casos, a polidispersidade dos polímeros obtidos do iniciador benzil-TEMPO aumenta gradualmente com o aumento da conversão. Pode ser visto também que a maioria das polidispersões está abaixo do valor esperado para uma terminação obtida por iniciador bimolecular ${ }^{[11]}$. Em geral, o monômero de p-acetoxiestireno exibe valores de polidispersão ligeiramente mais elevados ao longo da polimerização "viva" quando comparado ao estireno. As massas molares dos polímeros de p-acetoxiestireno, apresentados na Tabela 2, aumentam quase que linearmente com a conversão, indicando a ocorrência de polimerização "viva". Além disso, o controle moderado da polidispersão e o aumento da massa molar com tempo indicam que a natureza "viva" da polimerização não é afetada pela presença do grupo elétron-aceptor na posição para do estireno. Estes resultados estão de acordo com aqueles obtidos por Barclay e colaboradores $^{[11]}$.

\section{Copolímeros Diblocos}

Na Tabela 2 está sumarizada a caracterização das amostras através da cromatografia de permeação em gel (GPC) e calorimetria diferencial de varredura (DSC) junto com a composição química dos copolímeros em bloco.

As análises de GPC indicaram a formação de copolímeros em bloco com massas molares e polidispersões mais altas quando comparadas aos homopolímeros correspondentes. Os copolímeros de poli(p-acetoxiestireno-b-isopreno) foram preparados com bom controle da composição e com massa molar até 94500 u.m.a e a distribuição de massa molar foi inferior a 2,0. A análise de DSC mostrou que a temperatura de transição vítrea do bloco de poli(p-acetoxiestireno) apresentou o mesmo valor que a do homopolímero de p-acetoxiestireno $\left(100-120^{\circ} \mathrm{C}\right)$ relatado na literatura ${ }^{[11]}$, em- bora 5 a $18{ }^{\circ} \mathrm{C}$ mais baixa do que aqueles determinados para os homopolímeros correspondentes. A temperatura transição vítrea dos blocos de poli-isopreno teve valor similar àqueles dos homopolímeros de isopreno $\left(-67 \mathrm{a}-55^{\circ} \mathrm{C}\right)^{[15]}$. Nenhuma outra transição foi observada no DSC. Esses resultados de caracterização indicam que o copolímero em bloco de p-acetoxiestireno e isopreno foi obtido.

\section{Conclusões}

A síntese de vários poli(p-acetoxiestireno) (PASt) via um procedimento de polimerização radicalar "viva" foi demonstrada. Uma distribuição de massa molar moderada foi mantida ao longo da polimerização do p-acetoxiestireno. A natureza controlada deste sistema foi demonstrada por formação de copolímeros diblocos bem definidos. Em trabalho futuro será mostrada a avaliação da hidrólise destes homopolímeros e copolímeros diblocos.

\section{Agradecimentos}

Os autores agradecem a FAPESP (99/01783-0, 99/08444-6 e 01/12849-3) e ao CNPq (Projeto Milênio) pelo apoio financeiro.

\section{Referências Bibliográficas}

1. Rempp, P.; Franta, E.; Herz, J.; Adv. Polym. Sci., 86, 145 (1988).

2. Webster, O. W.; Science, 251, 887 (1991).

3. Hawker, C. J; Bosman, A. W.; Harth, E.; Chem. Rev., 101, 3661 (2001).

4. Benoit, D.; Harth, E.; Fox, P.; Waymouth, R. M.; Hawker, C. J.; Macromolecules, 33, 363 (2000).

5. Malmström, E. E.; Hawker, C. J.; Macromol. Chem. Phys., 199, 923 (1998). 
6. Georges, M. K.; Veregin, R. P. N.; Kasmaier, P. M.; Halmer, G. K.; Macromolecules, 26, 2987 (1993).

7. Aguiar, M; Hu, B; Karasz, F.E.; Akcelrud, L.; Macromolecules, 29, 3161 (1996).

8. Barclay, G. G.; King, M.; Sinta, R.; Malmström, E.; Ito, H.; Hawker, C. J.; Polym Prep., 38, 902 (1997).

9. Puskas, J. E.; Kaszas, G.; Prog. Polym. Sci., 25, 403 (2000).

10. Chen, X.; Jankova, K.; Kops, J.; Batsberg, W. J.; Polym. Sci. Polym. Chem. Ed., 37, 627 (1999).

11. Barclay, G. G.; Hawker, C, J.; Orellana, A.; Malefant, P. R. L.; Sinta, R. F.; Macromolecules, 31, 1024 (1998).
12. Chen, X. Y.; Iván, B.; Kops, J; Batsberg, W.; Macromol. Rapid Comm., 19, 585 (1998).

13. Gao, B.; Chen, X. Y.; Iván, B.; Kops, J.; Batsberg, W.; Macromol. Rapid Commun., 18, 1095 (1997).

14. Hawker, C. J.; Barclay, G. G.; Orellana, A.; Dao, J.; Devonport, W.; Macromolecules, 29, 5245 (1996).

15. Brandrup, J.; Immergut, E. H.; Polymer Handbook, Third Edition, (1989).

Enviado: 26/11/03

Reenviado: 06/04/04

Aprovado: 19/04/04 